\title{
Broadband Reflective Polarization Rotator Built on Single Substrate
}

\author{
Xiaofan Yang ${ }^{1}$, Tao $\mathrm{Qi}^{2}$, Yonghu Zeng ${ }^{1, *}$, Xiaoming $\mathrm{Liu}^{2,3, * \mathbb{C}}$, Gan Lu ${ }^{2,3}$ and Qing Cai ${ }^{4}$ \\ 1 State Key Laboratory of Complex Electromagnetic Environment Effects on Electronic and Information System, \\ Luoyang 471003, China; xiaofan_Uestc@sina.com \\ 2 School of Physics and Electronic Information, Anhui Normal University, Wuhu 241002, China; \\ wjxqt@ahnu.edu.cn (T.Q.); lgan@ahnu.edu.cn (G.L.) \\ 3 Anhui Provincial Engineering Laboratory on Information Fusion and Control of Intelligent Robot, \\ Wuhu 241002, China \\ 4 Shanghai Institute of Measurement and Testing Technology, Shanghai 201203, China; caiq@simt.com.cn \\ * Correspondence: zengyonghu@sina.com (Y.Z.); xiaoming.liu@ahnu.edu.cn (X.L.)
}

check for updates

Citation: Yang, X.; Qi, T.; Zeng, Y.; Liu, X.; Lu, G.; Cai, Q. Broadband Reflective Polarization Rotator Built on Single Substrate. Electronics 2021, 10, 916. https://doi.org/ 10.3390/electronics10080916

Academic Editors: Augustine O. Nwajana and Jiafeng Zhou

Received: 10 March 2021

Accepted: 9 April 2021

Published: 12 April 2021

Publisher's Note: MDPI stays neutral with regard to jurisdictional claims in published maps and institutional affiliations.

Copyright: (c) 2021 by the authors. Licensee MDPI, Basel, Switzerland. This article is an open access article distributed under the terms and conditions of the Creative Commons Attribution (CC BY) license (https:// creativecommons.org/licenses/by/ $4.0 /)$.

\begin{abstract}
A broadband polarization rotator built on single substrate is presented in this work. The device is designed for operation in the $\mathrm{K}$ and $\mathrm{Ka}$ bands. A slant array is used to achieve polarization rotation by $90^{\circ}$ in a reflective manner. Broadband has been obtained, with the operation frequency range covering $15-45 \mathrm{GHz}$ for $3 \mathrm{~dB}$ criteria, which is almost $100 \%$ fractional bandwidth. In addition, the insertion loss is less than $0.3 \mathrm{~dB}$ over a moderate broad incident angle from $0^{\circ}-20^{\circ}$. Furthermore, the polarization conversion ratio can be as high as 0.95 . By using a bi-static method, the fabricated prototype is measured, and the measured results demonstrate satisfactory agreement with the simulation ones. In comparison with other reflective designs in the literature, this design provides good bandwidth as well as polarization conversion ratio. Miniaturization can be investigated to increase the angular stability.
\end{abstract}

Keywords: polarization rotator; broadband; angular stable; reflective; frequency selective surface; slant array

\section{Introduction}

Polarization manipulation is a key function in a wide range of electronic systems. Polarization rotators are therefore employed in communication [1], radar detection [2] and radio astronomy [3]. A polarization rotator changes the polarization state of the incident wave into its orthogonal polarization direction, or rotates the linear polarization by $90^{\circ}$ [4] Such functionality can be realized by various techniques, such as Faraday rotator [5], roof-top mirror [6], and periodical structure [7]. Among these techniques, the periodical structure is one of the most popular methods owing to its flexibility in unit cell design and good performance [7-32].

Two types of polarization rotator can be made using periodical structures, i.e., the transmission type [7-15] and the reflection type [16-26]. The transmission type requires the incident wave travel through the whole structure to realize $90^{\circ}$ rotation. For the reflection type, the incident wave is reflected back to the same side with $90^{\circ}$ polarization rotation. It is found that polarization rotators working in the transmission type usually exhibit higher insertion loss and narrower band, in comparison with the reflection type. In light of this fact, the reflection type is preferred for low loss and wideband operation, and extensive investigation into this area has been conducted.

A split-ring and patch array was used to realize polarization rotation [16]. Such design was a typical way for creating $180^{\circ}$ phase difference so that the resultant polarization could be rotated [33]. This structure, however, exhibited limited bandwidth of $57.5 \%$. A very similar design used split rectangular ring (SRR) enclosed with a cross dipole was reported 
in [17]. The main drawback of this design is low polarization conversion ratio (PCR). Double-SRR structure was also investigated [18], however, the PCR was not improved considerably. Another modified design was based on dual-slit loop enclosed with a cross dipole, which did not produce better PCR or wider bandwidth [19]. Double-L unit cell was designed and two cascaded dielectric layers in [20]. The L-shaped strips were embedded in the dielectric layers to provide broadband operation. However, the fabrication will be a bit more complicated. A very interesting design using perforated array was reported [21]. The bandwidth can be as broad as $110 \%$ with satisfactory PCR. The main difficulty is that fabrication on dielectric with a large number of via holes. Based on the split circular ring, a switchable reflective design was realized [22]. The bandwidth of this structure is around 35\%, much narrower than other designs. A slant fractal array was employed to produce wideband polarization rotation with PCR as high as 0.9 [24]. This design is very instructive to create multi resonance in the passband. Three dimensional structures were also developed $[25,26]$. Although, these 3D designs are very interesting, the fabrication is difficult and no significant improvement has been observed in terms of bandwidth and PCR. There are many designs that have been reported, and the authors shall apologize to these contributors for not being able to include these works in this paper.

It is therefore worthy of further investigation to develop easy fabrication and wideband polarization rotator. In this paper, a reflective slant array is proposed to achieve polarization rotation. The unit cell is a slant strip, where the ends are loaded with stubs and the middle is loaded with patch. This is done to create multi resonance in the pass band, as well as to improve the in-band insertion loss. Such a structure is built on a single substrate, where the front layer is patterned with unit cells and the bottom layer is metal ground. Broadband has been realized, and good angular stability is also observed. It is demonstrated that such structure has very low insertion loss and very high PCR.

The remaining parts are organized as follows. Section 2 describes the design, optimization and parametric study; Section 3 presents measurement and results; and Section 4 concludes this work.

\section{Structure and Analysis}

For a train of $x$-polarized wave, when incident on the periodical array, the output wave will be $y$-polarized. The unit cell of the proposed wideband reflective polarization rotator is shown in Figure 1. To achieve polarization rotation, the periodical array is slantly arranged, and the unit cell consists of a capital I shape and a patch at the middle. The PTFE substrate is $1.5 \mathrm{~mm}$ thick. Additionally, the dielectric constant of the substrate is 2.65 . The back layer is a reflection metal plane. The parameters of the final design are shown in Table 1.

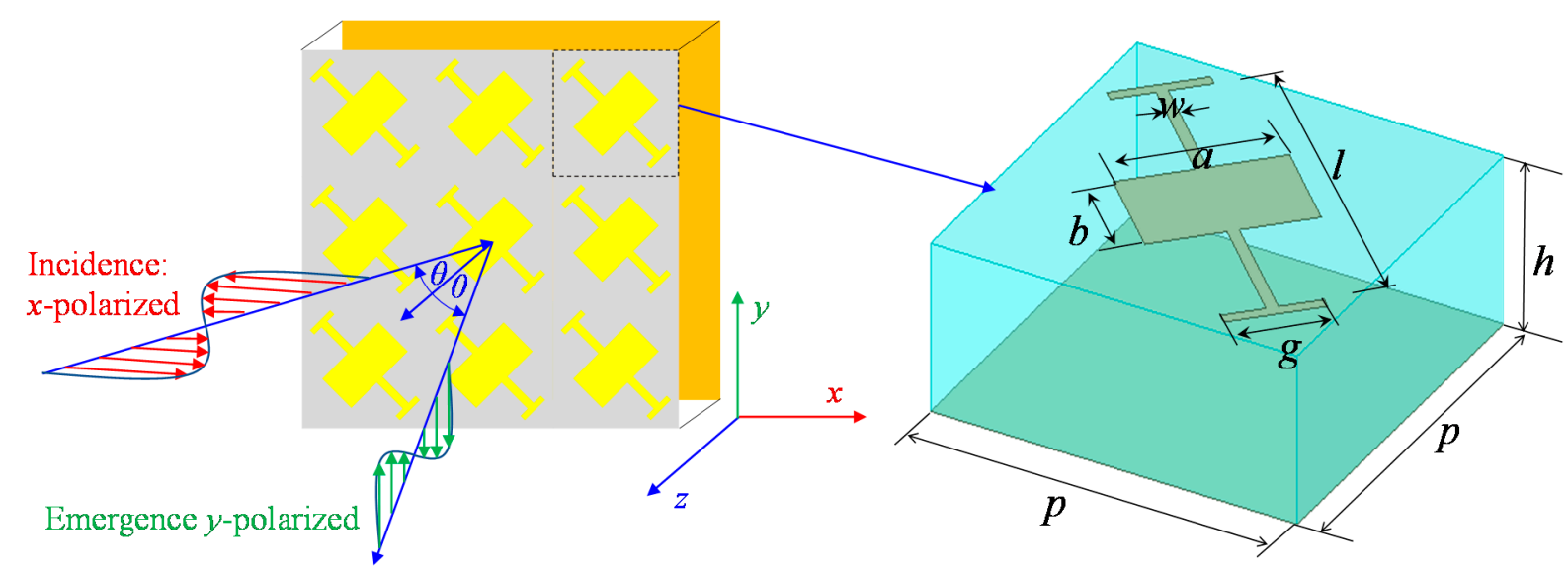

Figure 1. An illustration of the polarization rotator. Left panel: a demonstration of $x$-polarized wave incident on the periodical structure with emergence $y$-polarized wave; right panel: the unit cell of the polarization rotator. 
Table 1. The key parameters for the unit cell.

\begin{tabular}{lccccccc}
\hline Parameters & $\boldsymbol{p}$ & $\boldsymbol{a}$ & $\boldsymbol{b}$ & $\boldsymbol{w}$ & $\boldsymbol{g}$ & $\boldsymbol{h}$ & $\boldsymbol{l}$ \\
\hline Value $/ \mathrm{mm}$ & 3.9 & 1.7 & 1.1 & 0.12 & 1 & 1.5 & 3.8 \\
\hline
\end{tabular}

For a reflection type polarization rotator, the reflection coefficients are used to characterize the conversion. If the incident wave is $x$-polarized, the reflected wave may have two components, the $x$-component and the $y$-component. These components are characterized using $R_{x x}$ and $R_{y x}$, representing the ratios coupled to $x$-component and the $y$-component from the incident $x$-polarized wave. The simulation was conducted by using commercial software HFSS. By employing periodical boundary, only the unit cell is needed to predict the response of the polarization rotator. The simulated results are plotted in Figure 2, where three resonant frequencies $(18.2 \mathrm{GHz}, 27 \mathrm{GHz}, 39.4 \mathrm{GHz})$ are clearly observed. The $-3 \mathrm{~dB}$ bandwidth reads as $30 \mathrm{GHz}$ (from $15 \mathrm{GHz}$ to $45 \mathrm{GHz}$ ), showing a $100 \%$ fractional bandwidth. The insertion loss is very low, less than $0.3 \mathrm{~dB}$ in the central region of the pass band. It has to be mentioned that, since the structure is symmetrical for $x$-polarized and $y$-polarized waves, $R_{x y}$ is identical to $R_{y x}$ and $R_{x x}$ is identical to $R_{y y}$. In this connection, only $R_{y x}$ and $R_{x x}$ are plotted. The parametric study in coming part also shows that the variation in these coefficients is negligible within the fabrication accuracy. Therefore, the above observations can be made in the context of fabrication accuracy.
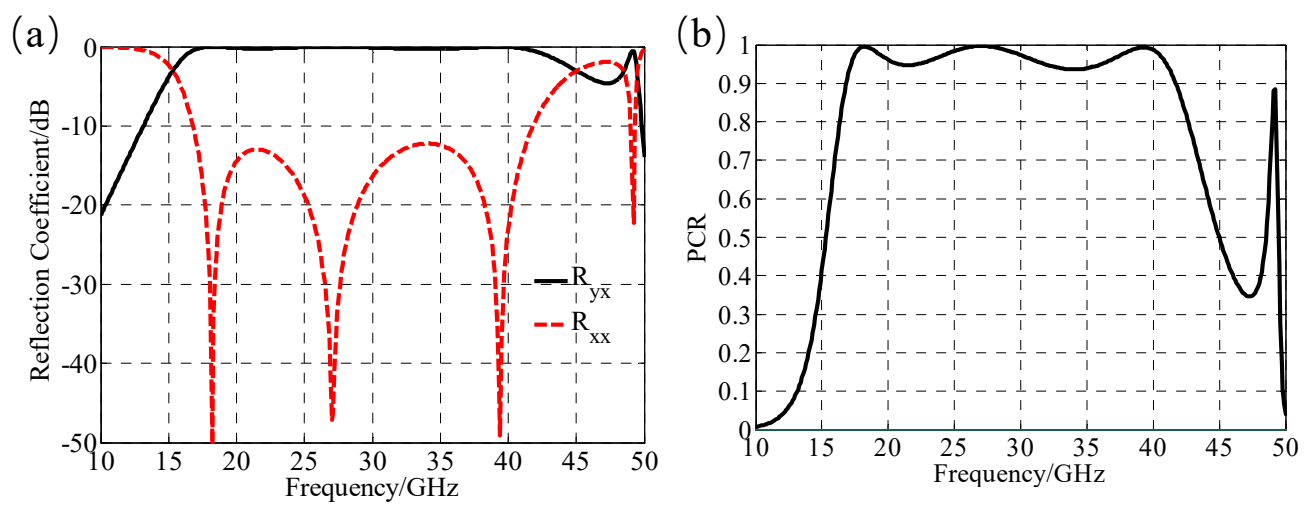

Figure 2. The simulated results. (a) The reflection coefficients in the range of $10-50 \mathrm{GHz}$; (b) the polarization conversion ratio.

The PCR can be calculated using [16]:

$$
\mathrm{PCR}=\frac{\left|R_{y x}\right|^{2}}{\left|R_{x x}\right|^{2}+\left|R_{y x}\right|^{2}}
$$

The PCR defines the fractional power of the $y$-polarized component in the reflected power. It is found that the majority region of the passband shows a PCR as high as 0.94.

The evolution of the design is presented in Figure 3a. Slant dipole array has been reported to be able to produce polarization rotation [32]. However, it is seen that single layer slant dipole array does not produce very good bandwidth. The end is then loaded to minimize the design, which is manifested by the shift of the first resonance to the lower frequency side. The loading, however, makes the in-band insertion loss worse, which is due to that the far distance between the first and the second resonances. Therefore, the middle of the dipole is loaded with a patch, which creates another resonance near $27 \mathrm{GHz}$. This design eventually reaches a good balance between bandwidth and in-band insertion loss. 
(a)

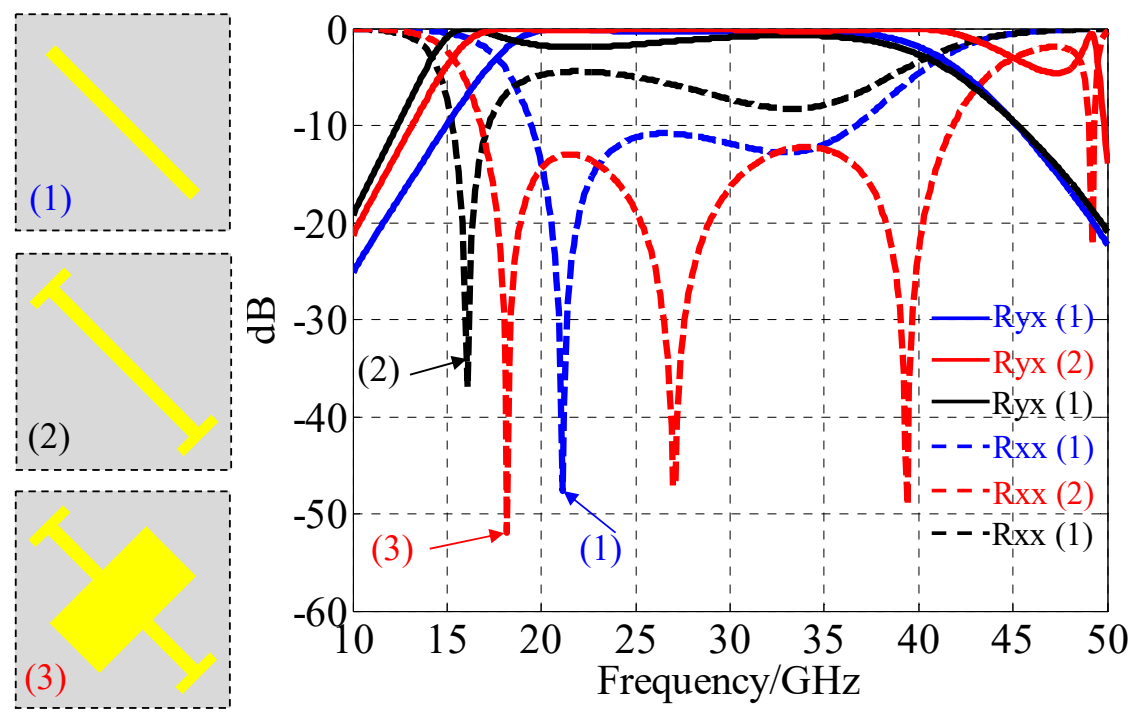

(b)
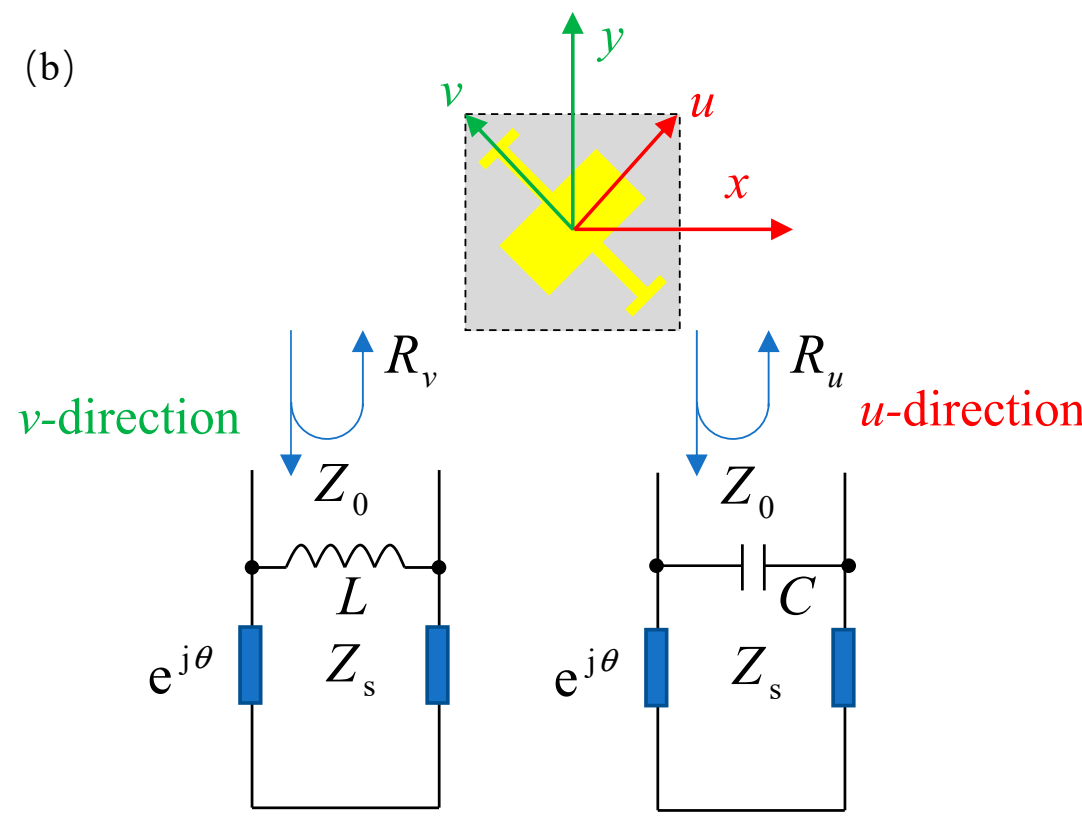

Figure 3. The evolution of the design and the equivalent circuit. (a) Evolution process: design (1) is a skewed strip array; design (2) is an end-loaded array; and design (3) is further loaded in the middle with a patch; (b) Equivalent circuit. The $v$-direction is an inductive effect; the $u$-direction is a capacitive effect.

An equivalent circuit for slant array can be described using Figure $3 b$, where the unit cell acts as an inductor to the $v$-direction, and a capacitor to the $u$-direction $[33,34]$. Therefore, the reflection coefficients can be written as:

$$
\left\{\begin{array}{l}
R_{y x}=\sqrt{(1-\cos \Delta \varphi) / 2} \\
R_{x x}=\sqrt{(1+\cos \Delta \varphi) / 2}
\end{array},\right.
$$

where $\Delta \varphi$ is the phase difference between $R_{v}$ and $R_{u}$ [33]. In this connection, for a polarization rotator, the phase difference $\Delta \varphi$ shall be $-180^{\circ}$.

To shed some light on the principle, the current distribution at the three resonate frequencies is plotted in Figure 4. The first resonance takes place at $18.2 \mathrm{GHz}$, where the 
length of the dipole (including the loaded stubs) corresponding to half wavelength in the substrate, which can be estimated using:

$$
\lambda_{\mathrm{g}}=\frac{\lambda_{0}}{\sqrt{\varepsilon_{\mathrm{eff}}}}=\frac{c}{f_{0} \sqrt{\varepsilon_{\mathrm{eff}}}}
$$

where $\varepsilon_{\text {eff }}$ is the effective dielectric [35]. The peak region on the bottom layer can be clearly recognized. For the second resonance at $27 \mathrm{GHz}$, it is clearly seen from the current distribution on the bottom layer that standing wave takes place. One peak and two valleys can be seen on the bottom layer. For the $39.4 \mathrm{GHz}$ resonance, the current is almost restricted to the patch region. The current distribution does verify that the patch creates more resonances in comparison to the loaded dipole.

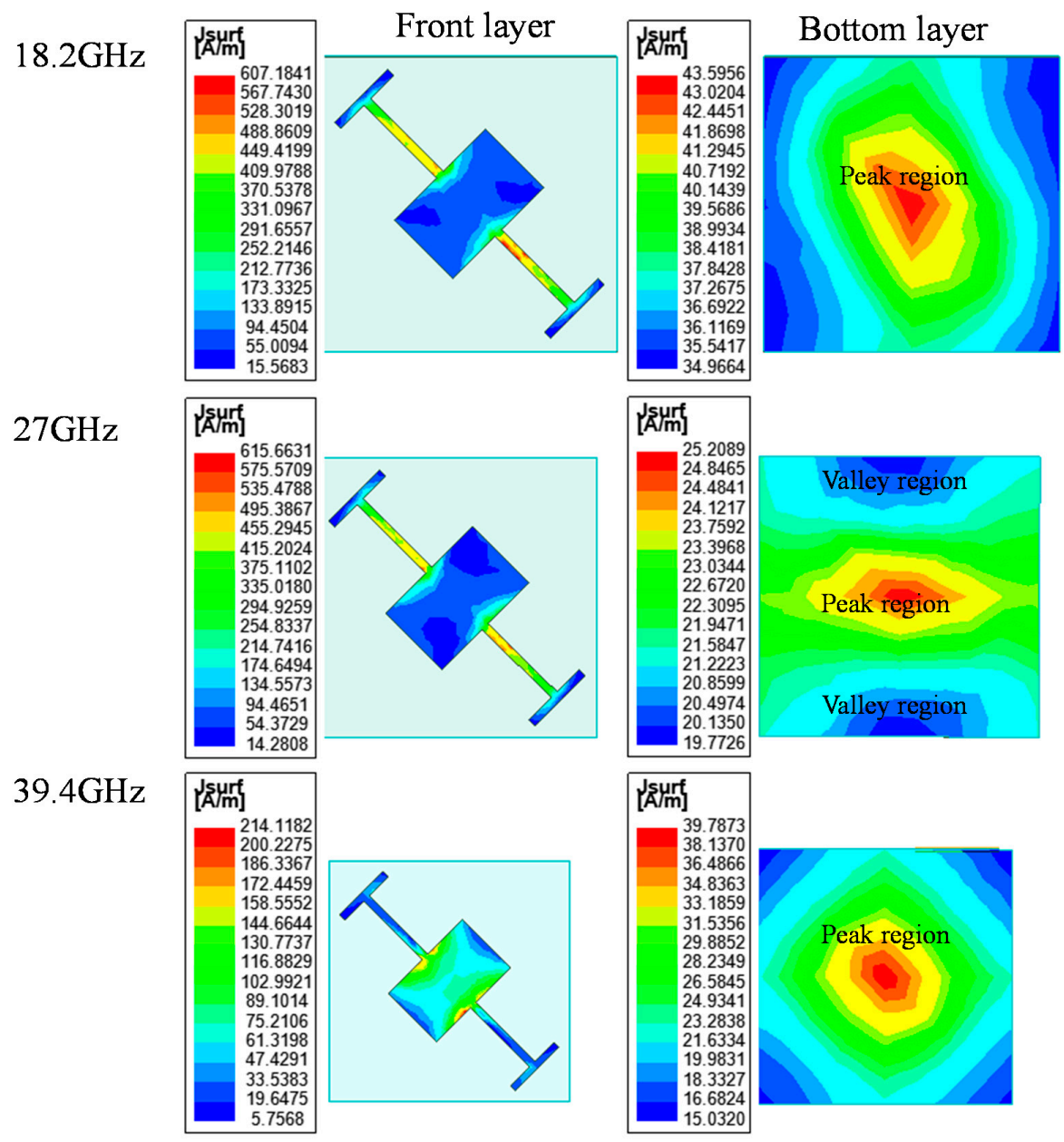

Figure 4. The current distribution at $18.2 \mathrm{GHz}$ (top row), $27 \mathrm{GHz}$ (middle row), and $39.4 \mathrm{GHz}$ (bottom row). The first column is for the front layer and the second column is for the bottom layer.

To investigate the sensitivity of each parameter, systematic parameter study has been conducted. The analysis is presented in Figure 5. Since the designed value for $w$ was only $120 \mu \mathrm{m}$, the step was set to $10 \mu \mathrm{m}$ (the fabrication accuracy), roughly $8 \%$ relative error to the designed value. A much larger step $(100 \mu \mathrm{m})$ was used for other parameters. This step would produce $10 \%$ error to the parameter $g$ (designed value $1 \mathrm{~mm}$ ), very close to the relative error of $w$. It is seen that the most sensitive parameter is the length of the unit cell $p$. And it is found that the second resonance is more liable to change of $p$. The length of the patch $a$ and the width $b$ also produce variation to the second resonance, however, is less influential in comparison to $p$. Other parameters are less sensitivity. These simulation results imply that $10 \mu \mathrm{m}$ fabrication accuracy is sufficiently good for this design. 
(a)

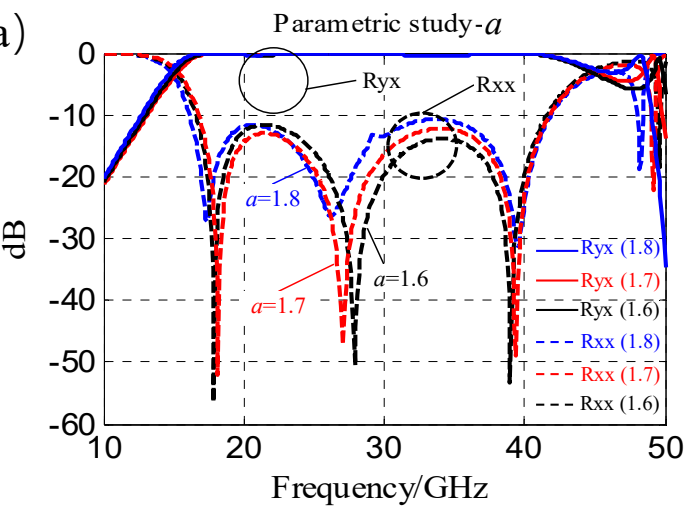

(c)

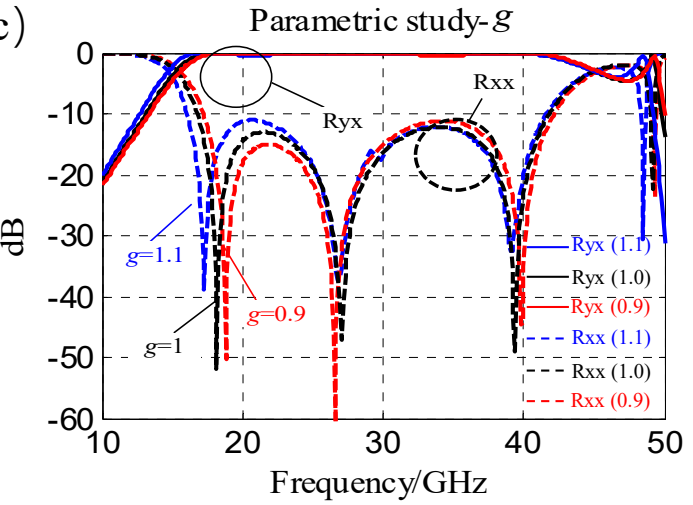

(e)

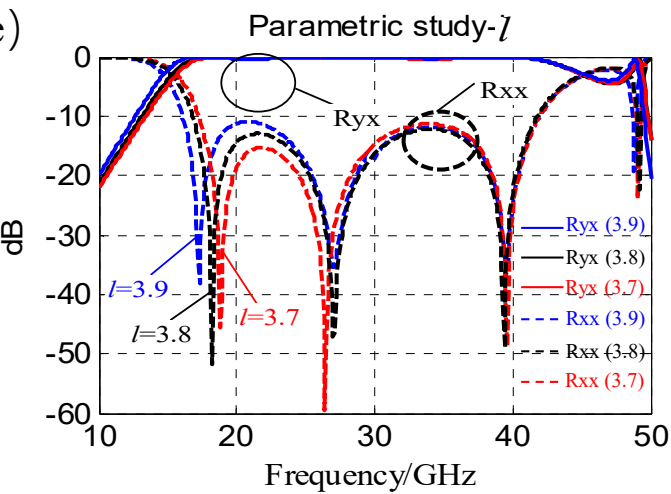

(g)

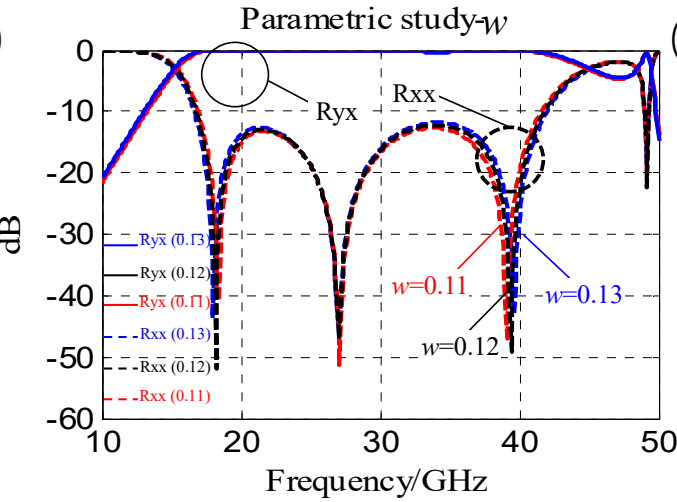

(b)
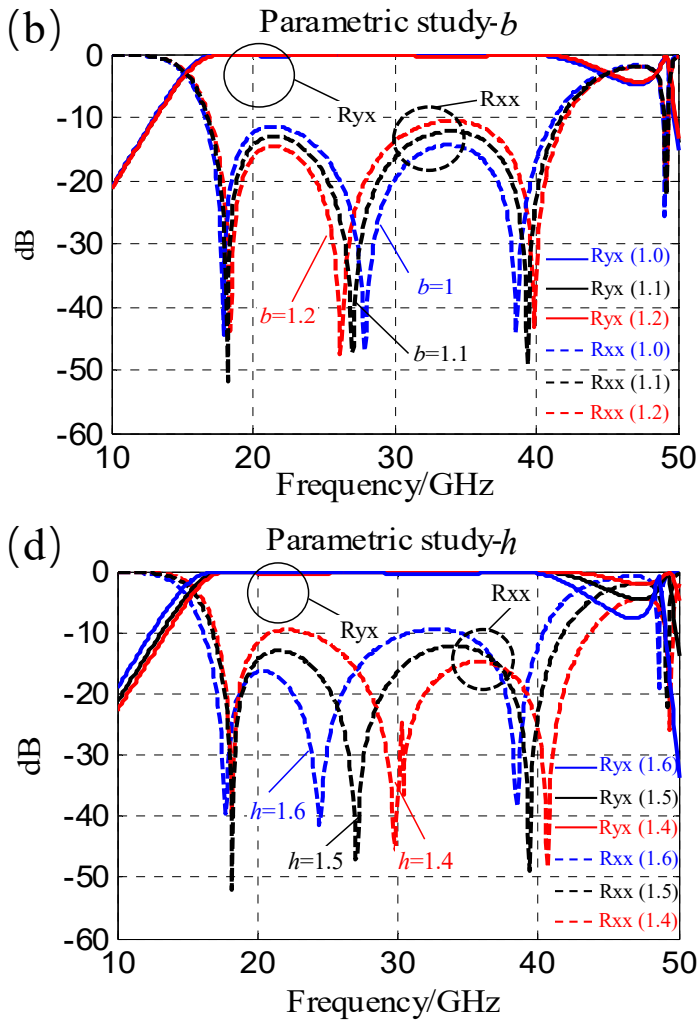

(f)

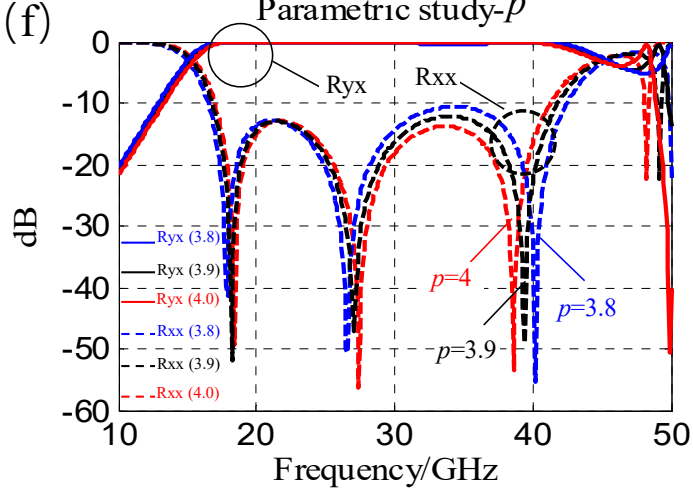

(h)

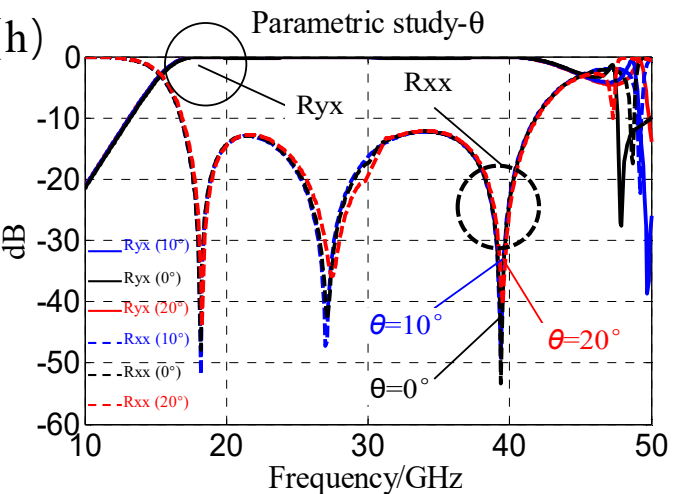

Figure 5. Parametric study for parameters (a) $a$, (b) $b,(\mathbf{c}) g,(\mathbf{d}) h,(\mathbf{e}) l,(\mathbf{f}) p,(\mathbf{g}) w,(\mathbf{h}) \theta$. The fabrication accuracy is $10 \mu \mathrm{m}$, the step is $10 \mu \mathrm{m}$ for $w$ and $100 \mu \mathrm{m}$ for other parameters.

\section{Fabrication and Measurement}

The fabricated polarization rotator is shown in Figure 6. The fabricated dimensions are $200 \mathrm{~mm}$ long and $200 \mathrm{~mm}$ wide. The substrate was first cut using a high-precision six-axis 
drilling/milling machine (HANS-F6M, Mason PCB, (Shenzhen, China)). Then, the pattern was etched out using the etching process. Surface quality control process was employed during fabrication. By using an industry microscope (SuperEyes, (Shenzhen, China)), the fabrication accuracy can be examined and measured after calibration. The key parameters are marked using the rule tool of the microscope. It can be seen that the fabrication accuracy is better than the expected value of $10 \mu \mathrm{m}$, showing satisfactory fabrication accuracy.

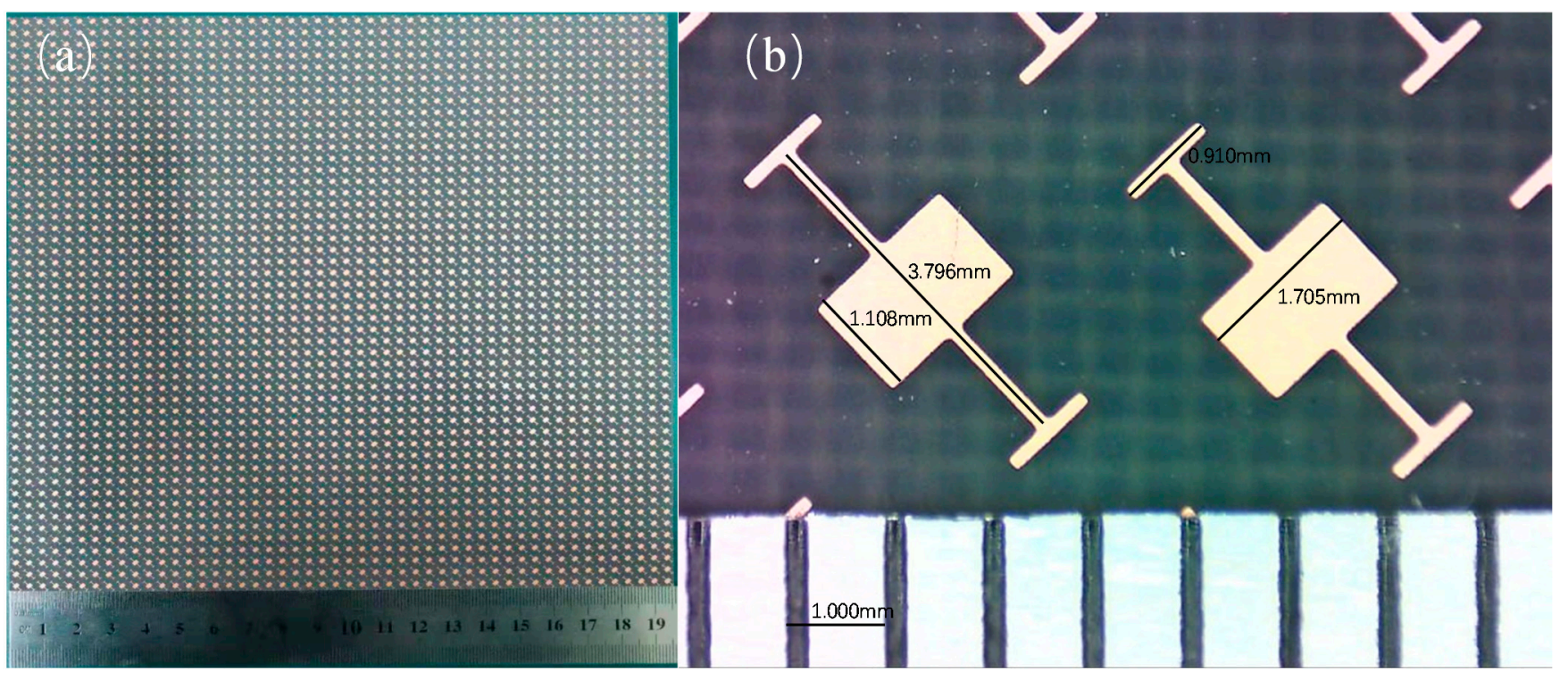

Figure 6. The fabricated prototype. (a) Photography in comparison with a ruler; (b) image under an industrial microscope with marked size.

Measurement was conducted using a free space method, as shown in Figure 7. The system was connected to a pair of broadband horns working from 12-40 GHz. A vector network analyzer (VNA, Cyear AV3276D, (Qingdao, China)) generated the input signal to the transmitting horn and collected the signal from the receiving horn. Radar absorption material (RAM) was mounted surrounding the sample to reduce scattering waves. In order to eliminate residual noise, time-gating technique was applied. The elevators were fixed on the wooden ground, and the horns were fixed on the top panel of the elevator. By averaging several rounds of measurement in combination with time-gating technique, the measured results demonstrated good repeatability. Due to the physical size of the horns, we only managed to measure the case of $\theta=20^{\circ}$. However, it is sufficient for the proof of concept.

The measurement procedures are as follows:

1. Background measurement on open air. The measurement was conducted by placing the bottom layer of the sample to the sample location so as to produce total reflection. This is due to that the bottom layer is the metal layer.

2. Recording the transmission coefficient of the VNA ( $\left.S_{21 \_a i r}\right)$. This was due to using the bi-static method to collect the reflected signal from the sample, as can be seen from Figure 7.

3. Measurement on sample for the reflection from $x$-polarization to $x$-polarization $R_{x x}$, and recording the transmission coefficient of the VNA $\left(S_{21 \_x x}\right)$.

4. Measurement on sample for the reflection from $x$-polarization to $y$-polarization $R_{y x}$, and recording the transmission coefficient of the VNA $\left(S_{21} y x\right)$.

5. Calculating the reflection coefficients by using $R_{x x}=S_{21 \_} x x-S_{21 \_a i r}$, and $R_{y x}=$ $S_{21 \_y x}-S_{21 \_a i r}$. This is done to remove the background effects. 
(a)

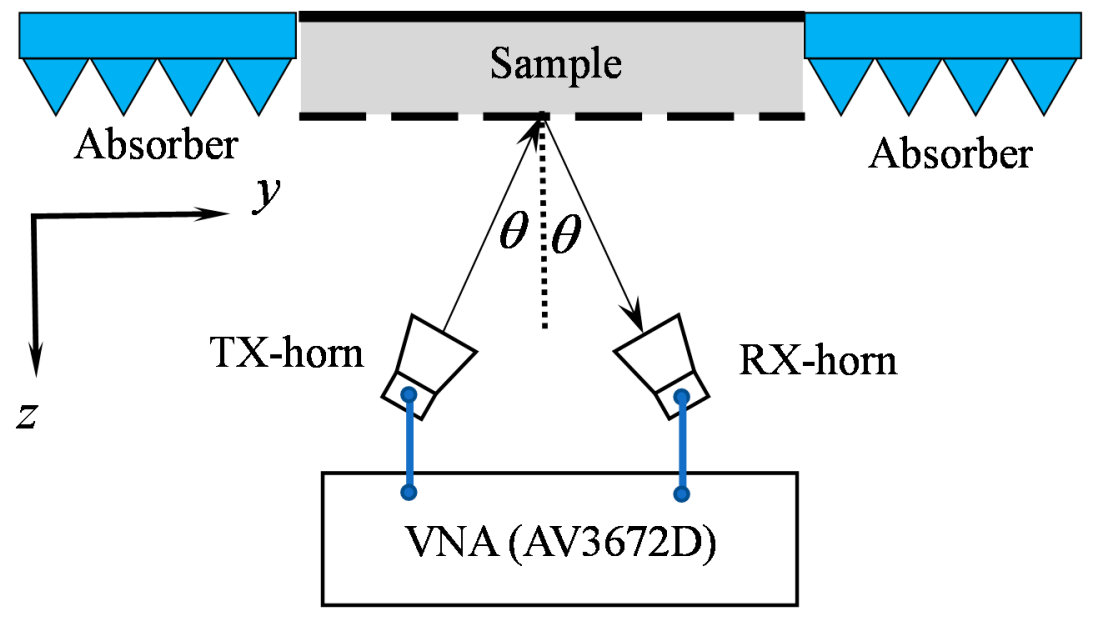

(b)

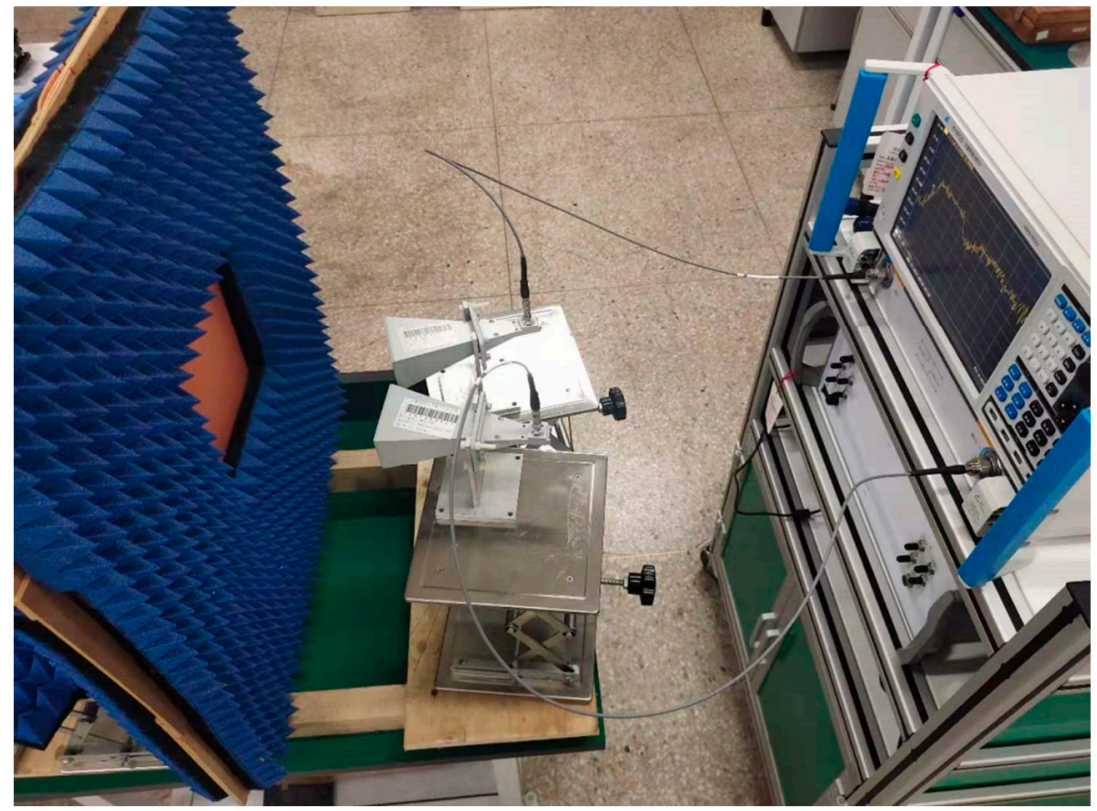

Figure 7. The measurement setup. (a) Schematic diagram using bistatic method; (b) instrumentation based on VNA technology.

The measured results are plotted in Figure 8. It is seen that the measured results are in good agreement with the simulation. For the reflection coefficients, the measurement was conducted from $12 \mathrm{GHz}$ to $40 \mathrm{GHz}$. The resonate frequencies agree with the simulation, only show slight frequency shift. The depth of resonance for the measure results are less than that of simulation. In addition, the measured PCR is also satisfactory good, larger than 0.92 in in most part of the passband. The discrepancy between the measurement and simulation may be attributed to that the PCB produces more loss than simulation. Larger loss in turn causes lower resonance and more insertion loss.

A comparison of this work with that in the literature is presented in Table 2. These selected designs are all of reflection type fabricated with no more than two layers. The comparison is made in regard to bandwidth, layers and realized PCR. It is seen that the bandwidth of structures made of two layers is wider than the design in this work. However, compared to single substrate, this work provides broadband operation, up to $100 \%$. Furthermore, the PCR is satisfactorily high, up to 0.95 by simulation, and better than 0.92 for measurement. Such results demonstrate that this design can be used for broadband polarization rotation with sufficiently good PCR. 

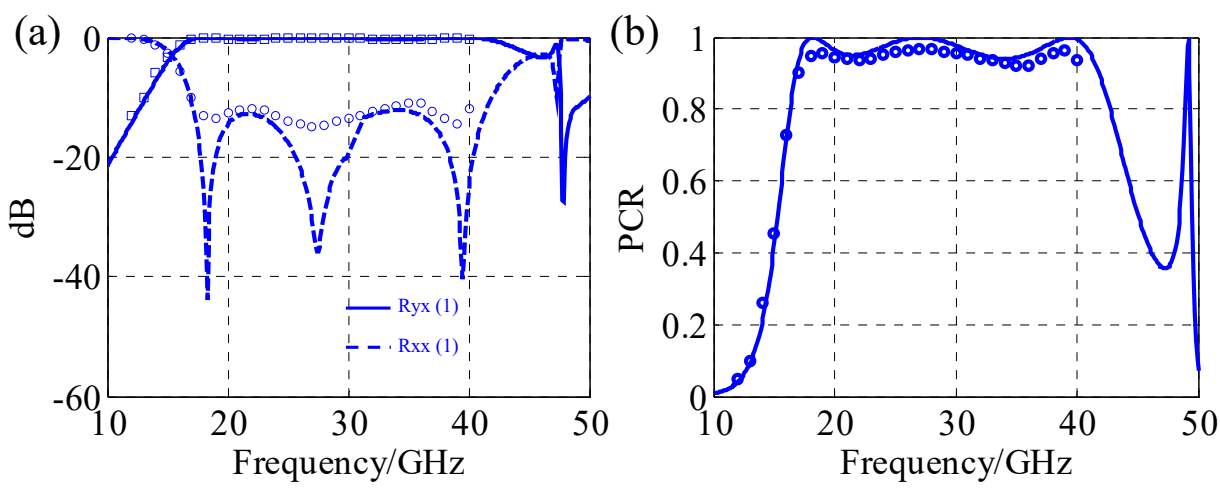

Figure 8. Comparison between measurement and simulation. (a) $R x x$ and $R y x$; (b) PCR. Measurement was conducted at $20^{\circ}$.

Table 2. A performance comparison of reflective polarization rotators presented in literature.

\begin{tabular}{ccccc}
\hline Ref. & Freq. (GHz) & 3 dB Bandwidth (\%) & Layers & PCR \\
\hline$[16]$ & $5.5-10.5$ & $62.5 \%$ & 1 & 0.88 \\
{$[17]$} & $5-9.7,11.2-15$ & $63.5 \%, 29 \%$ & 1 & 0.6 \\
{$[18]$} & $5-10.8$ & $73 \%$ & 1 & 0.6 \\
{$[19]$} & $5.6-11.4$ & $66 \%$ & 1 & 0.75 \\
{$[20]$} & $6-34$ & $140 \%$ & 2 & 0.8 \\
{$[21]$} & $15-55$ & $116 \%$ & 2 & 0.89 \\
{$[22]$} & $2.42-3.52$ & $37 \%$ & 2 & - \\
{$[23]$} & $8-18$ & $68.6 \%$ & 1 & 0.65 \\
{$[24]$} & $8-23$ & $100 \%$ & 1 & 0.9 \\
{$[25]$} & $4-6$ & $40 \%$ & 2 & 0.9 \\
{$[26]$} & X-band, Ku-band & $16.1 \%, 20 \%$ & 2 & 0.86 \\
This work & $15-45$ & $100 \%$ & 1 & 0.95 \\
\hline
\end{tabular}

Actually, to achieve wideband property, more resonances have to be created. The method used in [17] is a dual split-ring structure. Unfortunately, the PCR or bandwidth was not improved, though two resonances were created. The reason might be that the coupling between the two resonances was not balanced. The same issue is also found with [20], where the PCR is 0.8 over the passband. The perforated array is itself a multi-resonance structure, although the fabrication is a bit more complicated. In [24] the fractal structure is also a multi-resonance structure producing wideband operation with 0.9 PCR. Actually, the slant I shape is also capable of creating polarization rotation. By loading the patch at the middle, more resonances are induced. This is instructive to design wideband reflective polarization rotator by using multi-resonance structures. The only challenge is to increase the angular stability. One possible approach is to minimize the unit cell.

\section{Conclusions}

A broadband polarization rotator has been realized based on single substrate. The prototype was measured using the free-space method. The unit cell consists of an I-shaped dipole loaded at the center with a rectangular patch so as to create more resonance. The fractional $-3 \mathrm{~dB}$ bandwidth reads $100 \%$ and the measured PCR is better than 0.92 . Such a structure can be used as wideband polarization rotator with very high PCR. The design based on single substrate enable easy fabrication using common PCB materials. In regard to the angular stability, only $20^{\circ}$ is realized, and there still space for further improvement by using miniaturization methods. The bandwidth enhancement is also a good direction for further improvement. 
Author Contributions: Data curation: X.Y. and T.Q.; investigation: Y.Z. and X.L.; methodology: G.L. and Q.C.; resources: Y.Z.; writing—original draft: X.Y.; writing—review and editing: X.L. and Y.Z. All authors have read and agreed to the published version of the manuscript.

Funding: This work was supported by the National Natural Science Foundation of China (61871003), the State Key Laboratory of Complex Electromagnetic Environment Effects on Electronics and Information System (CEMEE2021Z0201B).

Conflicts of Interest: The authors declare no conflict of interest.

\section{References}

1. Sampath, S.S.; Sivasamy, R.; Kumar, K.J.J. A Novel Miniaturized Polarization Independent Band-Stop Frequency Selective Surface. IEEE Trans. Electromagn. Compat. 2019, 61, 1678-1681. [CrossRef]

2. Omar, A.A.; Shen, Z. Thin 3-D bandpass frequency-selective structure based on folded substrate for conformal radome applications. IEEE Trans. Antennas Propag. 2019, 67, 282-290. [CrossRef]

3. Chen, T.; Chen, X.; Yao, Y.; Liu, X.; Yu, J.A. Gaussian beam mode analysis method for 3-D multi-reflector quasi-optical systems. Electronics 2021, 10, 499. [CrossRef]

4. Pisano, G.; Melhuish, S.; Savini, G.; Piccirillo, L.; Maffei, B. A broadband W-band polarization rotator with very low cross polarization. IEEE Microw. Wirel. Compon. Lett. 2011, 21, 127-129. [CrossRef]

5. Erickson, N.R.; Grosslein, R.M. A low-loss 74-110-GHz Faraday polarization rotator. IEEE Trans. Microw. Theory Tech. 2007, 55, 2495-2501. [CrossRef]

6. Goldsmith, P.F. Quasioptical Systems: Gaussian Beam Quasioptical Propagation and Applications; IEEE Press: New York, NY, USA, 1998.

7. Liu, X.; Qi, T.; Wang, C.; Yang, X.; Gan, L.; Cai, Q. A broadband ultra-thin polarization rotator using periodically loaded parallel strip-lines. IEEE Access 2021, 9, 25450-25457. [CrossRef]

8. Wang, S.Y.; Liu, W.; Geyi, W. Dual-band transmission polarization converter based on planar-dipole pair frequency selective surface. Sci. Rep. 2018, 8, 1-9. [CrossRef] [PubMed]

9. Kanth, V.K.; Raghavan, S. Ultrathin design and implementation of planar and conformal polarization rotating frequency selective surface based on SIW technology. IET Microw. Anetennas Propag. 2018, 12, 1939-1947.

10. Saikia, M.; Ghosh, S.; Srivastava, K. Design and analysis of ultra-thin polarization rotating frequency selective surface using v-shaped slots. IEEE Antennas Wirel. Propag. Lett. 2007, 16, 2022-2025. [CrossRef]

11. Mollaei, S.M. Narrow-band configurable polarization rotator using frequency selective surface based on circular substrate integrated waveguide cavity. IEEE Antennas Wirel. Propag. Lett. 2017, 16, 1923-1926. [CrossRef]

12. Li, Y.; Zheng, L.; Xu, Z.; Zhang, J.; Wang, J.; Zhang, A.; Qu, S. Ultra-broadband linearly polarisation manipulation metamaterial. Electron. Lett. 2014, 50, 1658-1660. [CrossRef]

13. Mutlu, M.; Ozbay, E. A transparent $90^{\circ}$ polarization rotator by combining chirality and electromagnetic wave tunneling. Appl. Phys. Lett. 2012, 100, 051909. [CrossRef]

14. Arnieri, E.; Greco, F.; Boccia, L.; Amendola, G. A SIW-based polarization rotator with an application to linear-to-circular dual-band polarizers at K-/Ka-band. IEEE Trans. Antennas Propag. 2020, 68, 3730-3738. [CrossRef]

15. Garcia-Marin, E.; Masa-Campos, J.L.; Sanchez-Olivares, P.; Ruiz-Cruz, J.A. Bow-tie-shaped radiating element for single and dual circular polarization. IEEE Trans. Antennas Propag. 2020, 68, 754-764. [CrossRef]

16. Zhao, J.; Cheng, Y. A high-efficiency and broadband reflective $90^{\circ}$ linear polarization rotator based on anisotropic metamaterial. Appl. Phys. B 2016, 122, 1-7. [CrossRef]

17. Ye, Y.; He, S. $90^{\circ}$ polarization rotator using a bilayered chiral metamaterial with giant optical activity. Appl. Phys. Lett. 2010, 96, 203501. [CrossRef]

18. Khan, M.I.; Fraz, Q.; Tahir, F.A. Ultra-wideband cross polarization conversion metasurface insensitive to incidence angle. J. Appl. Phys. 2017, 121, 045103. [CrossRef]

19. Mustafa, M.E.; Izhar, R.; Wahidi, S.; Tahir, F.A. A broadband polarization rotator metasurface. In Proceedings of the 2019 International Conference on Microwave and Millimeter Wave Technology (ICMMT), Guangzhou, China, 19-22 May 2019.

20. Jia, Y.; Liu, Y.; Zhang, W.; Gong, S. Ultra-wideband and high-efficiency polarization rotator based on metasurface. Appl. Phys. Lett. 2016, 109, 051901. [CrossRef]

21. Omar, A.A.; Wonbin, H.; Amr, A.-A.; Abd-Elhady, M. A Single-Layer Vialess Wideband Reflective Polarization Rotator Utilizing Perforated Holes. IEEE Antennas Wirel. Propag. Lett. 2020, 19, 2053-2056. [CrossRef]

22. Saikia, M.; Ghosh, S.; Srivastava, K.V. Switchable reflective metamaterial polarisation rotator. Electron. Lett. 2016, 52, 1030-1032. [CrossRef]

23. Zhang, L.; Zhou, P.; Lu, H.; Chen, H.; Xie, J.; Deng, L. Ultra-thin reflective metamaterial polarization rotator based on multiple plasmon resonances. IEEE Antennas Wirel. Propag. Lett. 2015, 14, 1157-1160. [CrossRef]

24. Zheng, Q.; Guo, C.; Vandenbosch, G.A.E.; Yuan, P.; Ding, J. Ultra-broadband and high-efficiency reflective polarization rotator based on fractal metasurface with multiple plasmon resonances. Opt. Commun. 2019, 449, 73-78. [CrossRef] 
25. Cerveny, M.; Ford, K.L.; Tennant, A. Reflective switchable polarization rotator based on metasurface with PIN diodes. IEEE Trans. Antennas Propag. 2020, 68, 1483-1492.

26. Zhang, Z.; Luyen, H.; Booske, J.H.; Behdad, N. A dual-band, polarization-rotating reflectarray with independent phase control at each band. IEEE Trans. Antennas Propag. 2021. [CrossRef]

27. Yang, Y.; Wang, W.; Moitra, P.; Kravchenko, I.I.; Briggs, D.P.; Valentine, J. Dielectric meta-reflectarray for broadband linear polarization conversion and optical vortex generation. Nano Lett. 2014, 14, 1394-1399. [CrossRef]

28. Zhao, Y.; Cao, X.; Gao, J.; Liu, X.; Li, S. Jigsaw puzzle metasurface for multiple functions: Polarization conversion, anomalous reflection and diffusion. Opt. Express 2016, 24, 11208-11217. [CrossRef] [PubMed]

29. Torres, R.P.; Catedra, M.F. Analysis and design of a two-octave polarization rotator for microwave frequency. IEEE Trans. Antennas Propag. 2002, 41, 208-213. [CrossRef]

30. Huang, X.; Xiao, B.; Yang, D.; Yang, H. Ultra-broadband $90^{\circ}$ polarization rotator based on bi-anisotropic metamaterial. Opt. Commun. 2015, 338, 416-421. [CrossRef]

31. Zhu, X.C.; Hong, W.; Wu, K.; Tang, H.J.; Hao, Z.C.; Chen, J.X.; Zhou, H.X.; Hao, Z. Design of a bandwidth-enhanced polarization rotating frequency selective surface. IEEE Trans. Antennas Propag. 2014, 62, 940-944. [CrossRef]

32. Abdelmottaleb, O.A.; Shen, Z.; Yu, H.S. Multiband and wideband $90^{\circ}$ polarization rotators. IEEE Antennas Wirel. Propag. Lett. 2018, 17, 1822-1826.

33. Lin, B.; Wang, B.; Meng, W.; Da, X.; Li, L.; Fang, Y.; Zhu, Z. Dual-band high-efficiency polarization converter using an anisotropic metasurface. J. Appl. Phys. 2016, 119, 183103. [CrossRef]

34. Yu, H.; Su, J. Dual-Band and High-Efficiency Reflective Polarization Converter Based on Strip Grating. In Proceedings of the 2020 IEEE International Symposium on Antennas and Propagation and North American Radio Science Meeting, Montreal, QC, Canada, 5-10 July 2020. [CrossRef]

35. Pozar, D.M. Microwave Engineering, 4th ed.; Wiley: New York, NY, USA, 2012; Chapter 3. 